\title{
Interfacial Chemistry, Defects, and Strain in Multiferroic Heterostructures
}

\author{
J.D. Sloppy, ${ }^{*}$ J.C. Idrobo, ${ }^{* *}$ S. Sundaram , ${ }^{* * *}$ S.R. Spurgeon, ${ }^{*}$ C.R. W inkler, ${ }^{*}$ P. Munroe, ${ }^{* * *}$ N. \\ Valanoor, ${ }^{* * *}$ M.L. Taheri*
}

* Materials Science and Engine ering Department, Drexel Univ ersity, 3141 Chestnut Street, Philadelphia, PA 19104-2816

** Materials Science \& Technology Division, Oak Ridge National Laboratory

P.O. Box 2008, Oak Ridge, TN 37831-6069

*** School of Materials Science \& Engineering, The University of New South Wales, Sydney, NSW 2052, Australia.

The study of multiferroics is a burgeoning field of inquiry [1], partially owing to the hope of exploiting the magnetoelectric effect to permit rapid control of magnetic domain orientation via an applied electric field. Extrinsic multiferroic devices can be fabricated by layering ferroelectric and ferromagnet materials [2] whereby magnetoelectric coupling occurs via a strain-mediated mechanism. Magnetic properties of the structure are also dependent on strain. [3] Relationships between local chemistry, strain, and dislocation cores have been reported. [4] An improved understanding of strain-dependent coupling, interfacial chemistry, and misfit dislocations at the ferroelectric/ferromagnetic interface may allow for the development of models that predict device behavior and failure under an applied electric field.

In these studies, thin films of the ferromagnetic perovskite $\mathrm{La}_{0.67} \mathrm{Sr}_{0.33} \mathrm{MnO}$ (LSMO) are deposited on thin films of the ferroelectric perovskite $\mathrm{Pb}_{0.20} \mathrm{Zr}_{0.80} \mathrm{TiO}_{3}(\mathrm{PZT})$ via pulsed laser deposition (PLD). Figure 1 shows the deposition sequence of the structures. Strontium titanate $\left(\mathrm{SrTiO}_{3}\right)$ in the (100) orientation serve as the substrate and $\mathrm{SrRuO}_{3}$ is deposited as a conducting bottom electrode. The thickness of the PZT is varied between $20 \mathrm{~nm}$ and $50 \mathrm{~nm}$ while the thickness of the LSMO is constant. Top and bottom conducting electrodes allow for complementary in-situ biasing studies. [5] A HR-TEM image showing the LSMO/PZT and PZT/SRO interfaces is shown in Figure 2. The lattice constant of the PZT at the PZT/LSMO interface depends on the PZT thickness, thus the interfacial strain and the strain in the PZT and LSMO layers is varied by controlling the thickness of PZT.

Aberration-corrected, atomically-resolved STEM-EELS and Z-contrast imaging are applied to characterize the local chemistry and defect structure at the ferromagnetic/ferroelectric interface. Strain plays a vital role in determining the concentration and type of interfacial defects and is a crucial variable in defining the magnetoelectric coupling and exchange bias in the system as well as the nucleation, propagation, growth, and hysteresis behavior of ferromagnetic domains.

The ability to control magnetic orientation via magnetoelectric coupling may be doomed by high concentrations of misfit dislocations at the ferromagnetic/ferroelectric interface. Such an interface is anticipated to have a non-uniform electric field distribution. STEM-EELS allows for the electronic and chemical structure of dislocation cores to be studied at the atomic level and makes it possible to measure the diffusion of specific atoms in and around a dislocation core. The question of where oxygen atoms sit in relation to a dislocation core and how cations and oxygen vacancies distribute 
may ultimately determine the device properties. A better understanding of the phenomena and structure at the interface between ferroelectric and ferromagnetic materials is reported.

\section{References}

[1] L.W. Martin, et.al., Mat. Sci. Eng. R. 68 (2010) 891.

[2] R. Ramesh and N.A. Spaldin, Nat. Mat. 6 (2007) 21.

[3] J.L. Maurice, et.al., Phil. Mag. 83 (2003) 3201.

[4] Arredondo, et.al. Adv.Mat. 22 (2010) 1.

[5] C.R. Winkler, et.al., Microsc. Microanal. (2010), Portland, OR, USA.

[6] This research was supported by the Office of Naval Research under contract number N000141110296. (Program Manager Daniel Green) The as sistance of Dr. Crai g Johnson of Drexel University and his maintenance of TEM facilities is gratefully acknowledged.

\begin{tabular}{|c|c|}
\hline A & Hectode \\
\hline$L_{0.67} \mathrm{Sr}_{0.33} \mathrm{MnO}_{3}$ & Ferromagnetic \\
\hline $\mathrm{Pb}_{0.2} \mathrm{Zr}_{0.8} \mathrm{TO}_{3}$ & Ferroelectric \\
\hline SRO & Electrode \\
\hline STO (100) & Substrate \\
\hline
\end{tabular}

Figure 1: Schematic of multiferroic thin film structure fabricated by pulsed laser deposition on STO (100) substrate.

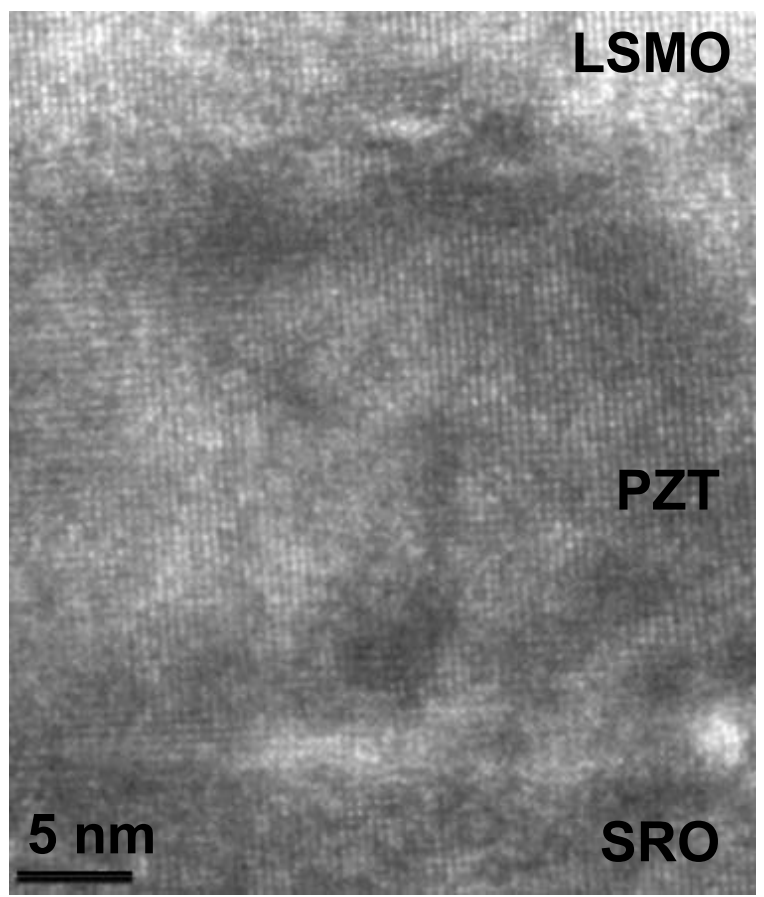

Figure 2: $\mathrm{HR}-\mathrm{TEM}$ image of $\mathrm{La}_{0.67} \mathrm{Sr}_{0.33} \mathrm{MnO}$ (LSMO) deposited on $\mathrm{Pb}_{0.20} \mathrm{Zr}_{0.80} \mathrm{TiO}_{3}(\mathrm{PZT})$. 